\title{
Facteurs d'ancrage territorial des activités agricoles. Soja et élevage en Argentine
}

Territorial Anchorage of Agricultural Activities: soybean and livestock in Argentine

Julio H. Elverdín, Andrea Maggio et José Muchnik

\section{(2) OpenEdition}

\section{Journals}

Édition électronique

URL : http://journals.openedition.org/economierurale/3008

DOI : 10.4000/economierurale.3008

ISSN : 2105-2581

\section{Éditeur}

Société Française d'Économie Rurale (SFER)

Édition imprimée

Date de publication : 21 mars 2011

Pagination : 64-78

ISSN : 0013-0559

Référence électronique

Julio H. Elverdín, Andrea Maggio et José Muchnik, « Facteurs d'ancrage territorial des activités agricoles. Soja et élevage en Argentine », Économie rurale [En ligne], 322 | mars-avril 2011, mis en ligne le 30 mars 2013, consulté le 19 avril 2019. URL : http://journals.openedition.org/economierurale/3008 ; DOI : 10.4000/economierurale.3008 


\section{Facteurs d'ancrage territorial des activités agricoles Soja et élevage en Argentine}

Julio H. ELVERDíN • Laboratoire AGRITERRIS, Instituto Nacional de Tecnología Agropecuaria (INTA), Estación Experimental Agropecuaria Balcarce, Argentine

Andrea MAGGIO • Instituto Nacional de Tecnología Agropecuaria (INTA), Argentine José MUCHNIK •INRA SAD / UMR Innovation, Montpellier

\section{Introduction}

La croissante volatilité du prix mondial des matières premières alimentaires à partir de l'année 2006 et le retour début 2008 des révoltes contre la faim, dans différents pays d'Afrique, d'Asie et d'Amérique Latine, obligent à reconsidérer l'importance de l'analyse des processus de localisation/délocalisation des activités agricoles dans la conjoncture actuelle. Il s'agit donc de processus qui sont étroitement associés à trois défis majeurs : (i) la sécurité alimentaire des populations; (ii) les nouveaux défis environnementaux ; (iii) l'évolution des territoires et des sociétés rurales, et leurs rapports aux dynamiques urbaines.

Les changements intervenus dans la production agricole argentine, ainsi que le « conflit agraire » témoignent bien des tensions et des tendances constatées au plan international, à tel point que l'on pourrait affirmer qu'il s'agit bien d'un cas exemplaire de cette évolution. L'industrialisation de l'agriculture, avec notamment l'expansion du soja, la profondeur des changements technologiques, la perte relative de rentabilité de l'élevage, l'impact sur les sociétés rurales, les modifications environnementales qui s'en sont suivies, les politiques de l'État visant la protection des consommateurs de la hausse des prix internationaux, sont autant de facteurs qui montrent que nous sommes face à une modification de type structurel, avec des conséquences à long terme.

L'envergure des changements a entraîné un foisonnement d'études et de publications, dont la plupart se focalisent sur l'interprétation des processus à partir d'une analyse « macro ». Rares sont par contre les travaux qui partent d'observations précises de terrain, de l'analyse des stratégies des producteurs et des évolutions territoriales. Il s'agit là de la principale contribution de cet article, car à partir d'enquêtes et d'observations faites au niveau local se pose la question : quelles sont les différentes stratégies des producteurs face aux changements technologiques et aux évolutions du marché, et comment affectent-elles l'ancrage territorial des activités de production?

Pour répondre à cette question nous nous pencherons sur :

(i) les processus de localisation/délocalisation des activités d'un point de vue historique et de leurs caractéristiques actuelles ;

(ii) la diversité des stratégies des producteurs face à ces changements, à partir de l'évolution constatée dans la localité de Bolívar de la Province de Buenos Aires où le processus d'industrialisation agricole/déplacement de l'élevage est intensif ;

(iii) les dynamiques territoriales à l'œuvre dans cette localité.

\section{Les processus de localisation/délocalisation des activités de production}

\section{L'ancrage territorial Un processus complexe}

La formulation « ancrage territorial » nous interroge sur les variables qui déterminent 
RECHERCHES

Julio H. ELVERDÍN, Andrea MAGGIO, José MUCHNIK

qu'une activité productive soit ancrée quelque part en signifiant en même temps que cette activité puisse lever l'ancre, se déplacer ailleurs. Un regard rétrospectif nous permet d'apprécier que les hommes et leurs savoirs, ainsi que les espèces végétales ou animales et les produits qui en dérivent, n'ont jamais cessé de voyager (MoityMaïzi et Muchnik 2005).

C'est d'ailleurs dans ces voyages incessants que l'on retrouve l'origine de la célèbre viande argentine (Giberti, 1981). En effet, les bovins sont arrivés d'Europe, se sont acclimatés et se sont reproduits librement - rappelons que jusqu'au XVIII ${ }^{\mathrm{e}}$ siècle il existait des permis de chasse des bovins. Des savoirs, des techniques d'élevage et d'engraissement, de gestion de pâtures et de troupeaux, ainsi que des cultures culinaires, qui aujourd'hui font partie des références identitaires argentines, se sont développés pour construire petit à petit la typicité des viandes argentines et l'ancrage territorial de l'activité d'élevage (Champredonde, 2008).

Les liens au lieu varient selon les individus et le type d'activité productive, conditionnant ainsi le degré d'ancrage territorial. L'attachement, non seulement économique mais également affectif et symbolique, à un lieu constitue un facteur important dans les processus de localisation/délocalisation. Or, l'ancrage territorial ne dépend pas que du lien aux lieux des acteurs concernés, il dépend également de l'évolution de l'environnement (économique, financier, technologique...) et des politiques publiques qui régulent les dynamiques territoriales. C'està-dire qu'il se rattache aux décisions prises par des acteurs différents dans des espaces différents, ce que nous représentons dans le schéma ci-dessous.

Le processus de diffusion de la culture du soja est exemplaire à ce propos. Originaire de l'Asie, le soja, destiné à la production d'huile et de tourteau, a connu pendant les trois dernières décennies une expansion accélérée aux États-Unis, au Brésil, en Argentine et en Europe. Ainsi, la culture du soja en Argentine est passée de 100000 hectares cultivés dans la période 1970-1973 à 17 millions d'hectares dans la campagne 2007/2008 (MAGyP, 2010). S'il est difficile d'en prédire l'évolution à long terme, nous pouvons toutefois constater qu'il s'agit d'une production avec un très faible ancrage territorial, tant du point de vue matériel génétique employé que des systèmes de gestion, des techniques

Schéma 1. Variables intervenant dans les processus d'ancrage/désancrage territoriaux des activités productives

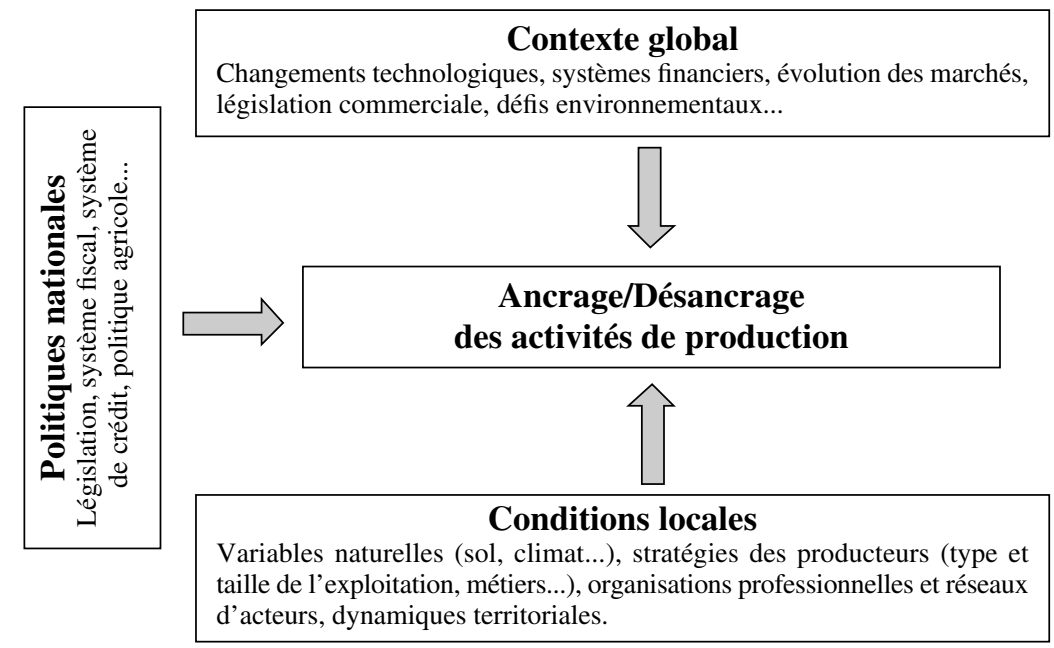

Source : les auteurs 
de production ou des cultures alimentaires associées. Par conséquent, les risques de délocalisation à la suite de changements de contexte sont élevés. Une stratégie à moyen et à long terme doit prévoir le degré d'irréversibilité de cette évolution ainsi que ses conséquences, de manière à développer des alternatives mieux adaptées et plus résilientes face à d'éventuels changements soudains du contexte.

\section{Des ordinateurs et des vaches Mondialisation et délocalisations}

Le processus de mondialisation dans l'univers de la gestion et de la finance implique des mécanismes de segmentation ou de « modularisation » dans le domaine technique et productif (Berger, 2006). On en retrouve des exemples typiques dans certains secteurs industriels (informatique, textile), où les grandes sociétés se consacrent à l'assemblage de composantes de leurs produits fabriquées dans différents points de la planète, alors que la maîtrise de la conception, du design et de la marque restent des éléments clés qui permettent à la « maison mère » d'assurer le contrôle sur l'ensemble.

La question qu'il faut se poser est si la filière agricole possède des différences qualitatives justifiant un traitement différencié par rapport à d'autres secteurs de production. De manière intuitive, il semble évident qu'il existe une différence qualitative entre la délocalisation de la production d'ordinateurs ou de chemises et celle de la production de viande ou de vin. D'un point de vue conceptuel, deux grands types de différences peuvent être identifiés :

(i) la filière agricole implique des rapports spécifiques à l'utilisation de la terre et à la gestion des ressources naturelles, des problématiques qui vont au-delà des

1. Le mot « résilience» vient du terme latin « resilio » qui signifie sauter en arrière, ressauter, rebondir. D'après S. Vanistendael et J. Lecomte (2000) le concept inclut également la capacité d'une personne ou d'un système social à surmonter correctement des difficultés. considérations strictement économiques et financières (la biodiversité, les paysages, la vie des sociétés rurales, les équilibres et les rapports entre la campagne et la ville) ;

(ii) les denrées alimentaires occupent une place à part vis-à-vis des autres biens de consommation, car il s'agit des seuls biens qui sont «in-corporés » (au sens étymologique : ils sont introduits dans le corps) (Fischler, 1990).

La santé des consommateurs, leur identité culturelle, les dynamiques des territoires qu'ils habitent, sont directement mises en cause au moment de la consommation d'aliments. C'est dans ce sens que nous caractérisons le fait alimentaire comme étant un « fait social total » (Mauss, 1935), c'est-àdire, un fait qui met en cause un ensemble de rapports sociaux.

On ne s'étonnera donc pas des tensions provoquées en Argentine par les conséquences de l'expansion de la culture du soja (figure 1) et du déplacement relatif de la production issue de l'élevage, car il s'agit de phénomènes qui vont bien au-delà du secteur agricole.

Ce n'est pas non plus par hasard si le contexte actuel donne lieu à des tensions similaires dans d'autres pays ou si les secteurs agricoles et agroalimentaires restent toujours le nœud gordien des négociations de l' Organisation mondiale du commerce (OMC) ou de l'Union européenne, dans la mesure où il s'agit de filières qui nécessitent des réglementations politiques spécifiques, depuis l'organisation et l'évolution des territoires ruraux jusqu'à l'alimentation, qualitative et quantitative, des populations. Ces réglementations conditionnent alors l'évolution de l'ensemble de la société.

\section{Aliments et territoires À la recherche de I'homus mundialis}

Le processus de mondialisation est complexe et traduit une double tendance. D'une part, on retrouve des activités, des 
Figure 1. Évolution de la surface semée des principales cultures au niveau national

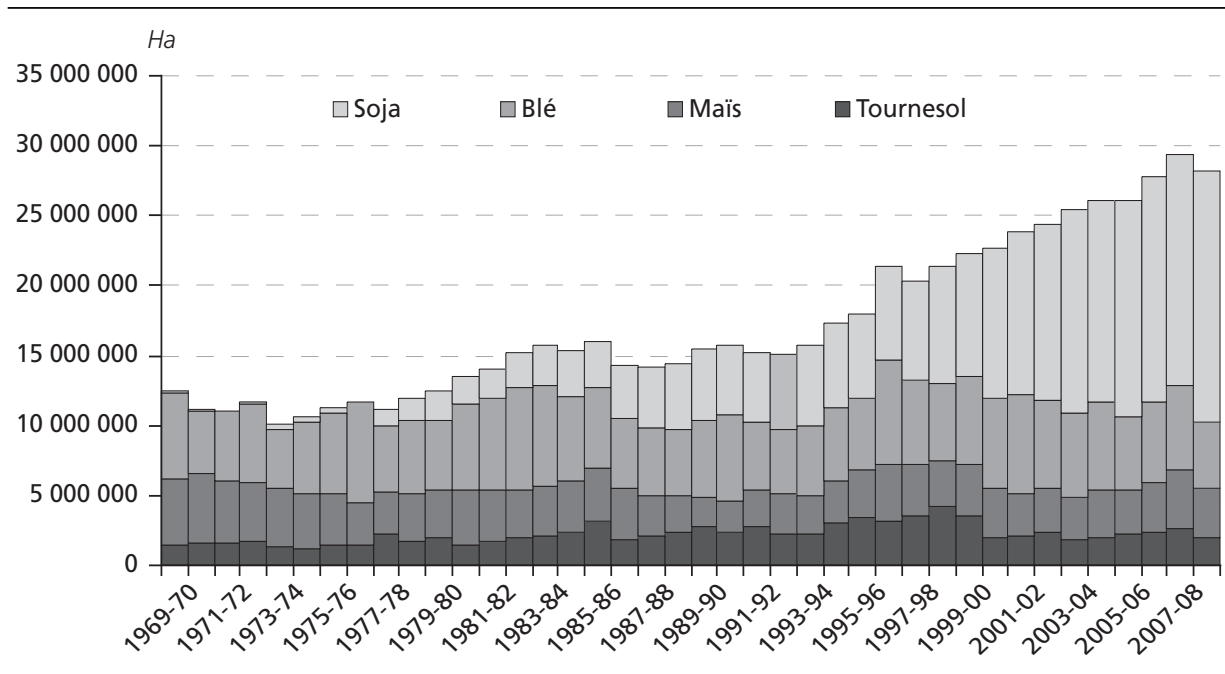

Source : Ministerio de Agricultura, Ganadería y Pesca de la Nación, 2010

technologies et des produits standardisés : nous pouvons acheter le même litre d'huile ou la même boîte de bière dans différents endroits de la planète. D'autre part, les hommes restent liés à des sociétés, des territoires, des cultures, des valeurs, qui donnent un sens à leurs vies et à leurs actes quotidiens (Muchnik et al., 2008), si bien qu'il devient difficile de trouver l'homus mundialis. C'est en raison du rôle particulier que jouent les denrées alimentaires, dans la construction des références identitaires, qu'il s'agit d'un domaine où la tendance anti-uniformisation se fait notamment sentir. Les consommateurs cherchent aussi des produits ayant des spécificités territoriales et des charges symboliques fortes, qui soient également associés à une mémoire gustative culturellement significative : les individus cherchent à " être et se sentir de quelque part» (Tizon, 1996).

Le processus d'expansion du soja et le recul de l'élevage peuvent également être lus à partir de cette perspective. Le courant principal de ce phénomène reste aujourd'hui évident, et il est clair que la rentabilité économique à court terme en a été la variable fondamentale. Or, l'enjeu de la concurrence entre le soja et l'élevage ne concerne pas que la rentabilité économique. Les identités et les métiers des producteurs, la vie dans les territoires ruraux, les cultures alimentaires, sont également en jeu. Reste à savoir dans quelle mesure ces facteurs peuvent contribuer au développement d'une contre-tendance et à l'établissement d'équilibres nouveaux.

D'un point de vue scientifique, il ne s'agit pas de formuler des jugements de valeur ou idéologiques, mais d'analyser les mécanismes à l'œuvre dans ce processus, ainsi que leurs conséquences au niveau environnemental, économique, social et alimentaire. Il s'agit également d'envisager des approches, des méthodologies et des outils destinés à développer des politiques et des stratégies à moyen et à long terme.

\section{Industrialisation agricole Expansion du soja et déplacement de l'élevage}

\section{L'évolution en chiffres dans la province de Buenos Aires}

Étant donné l'importance socio-économique du secteur agricole dans la province de Buenos Aires, ces chiffres sont représentatifs de l'évolution dans l'ensemble de la région pampéenne.

Entre 1988 et 2008, cette province affiche une diminution significative $(-58 \%)$ du 
Tableau 1. Nombre d'EAPs et surfaces dans la province de Buenos Aires

\begin{tabular}{l|c|c|c}
\hline Province de Buenos-Aires & Total EAPs & Surface Totale & Surface Moyenne \\
\hline CNA 1988 & 75531,00 & 27282510,10 & 361,21 \\
\hline CNA 2002 & 50956,00 & 25688253,00 & 504,13 \\
\hline CNA 2008 (estimation) & 31753,00 & 17445762,00 & 549,42 \\
\hline
\end{tabular}

Source : INDEC : CNA 1988, 2002 et 2008

nombre d'exploitations agricoles (EAPs) ${ }^{2}$, notamment dans le segment des plus petites exploitations (tableau 1). Au cours de la même période, la superficie moyenne par EAP augmente de 361 ha à 549 ha $(+52 \%)$.

Cette diminution du nombre d'EAPs, ainsi que la concentration des exploitations phénomènes généralisés dans la région de la Pampa - ont des effets sur le dépeuplement du secteur rural, et des conséquences sur les dynamiques territoriales qui se traduisent par la fragilisation et l'éclatement des réseaux sociaux traditionnels ainsi que par l'affaiblissement des services de base (éducation, santé, électricité - déjà insuffisants).

Par ailleurs il est intéressant d'analyser l'évolution des principales productions dans la province de Buenos Aires, qui montre bien l'expansion de la culture du soja et la diminution de l'élevage (figures 2 et 3), en particulier les cinq dernières années. Précisons que l'élevage bovin se développe dans des superficies réduites en faisant appel à des compléments alimentaires en remplacement du pâturage extensif, libérant de cette manière des superficies pour l'agriculture.

\section{L'industrialisation agricole : le " tout-soja "}

Parmi les cultures, celle du soja par semis direct est encouragée, notamment pour trois raisons :

- c'est la culture qui implique le plus faible investissement par hectare (Tosi, 2003, 2007 b) ;

2. Unité statistique des recensements nationaux agricoles (CNA) de 1988 et de 2002, en tant qu'unité de direction et de gestion de la production. Manuel du recenseur, CNA 1988.
- l'évolution favorable des prix par rapport à d'autres produits ;

- la simplification de la gestion de la technologie « soja transgénique par semis direct » (STSD). À partir de l'analyse économique $\mathrm{du}$ fonctionnement de ces systèmes de production dans la région pendant les dix dernières années (Tosi, 2007 a) on peut conclure que la marge brute des activités agricoles dépasse celle de l'élevage d'au moins $300 \%$ et davantage encore quand on se penche sur le retour sur investissements. De même, l'auteur signale des coûts directs plus faibles, ainsi que des marges brutes et des retours sur investissements plus importants pour le soja et le tournesol, par rapport au blé et au maïs.

Le fonctionnement du paquet technologique «Soja transgénique par semis direct $(S T S D) »$ repose fondamentalement sur :

- La possibilité de disposer de graines de soja génétiquement modifiées par l'incorporation du gène $\mathrm{RR}$ 《 Roundup Ready ${ }^{\circledR}$ » (résistance à l'herbicide à base de glyphosate $^{3}$ ) et adaptées à la zone en termes de durée du cycle de production de la culture. - Le stockage de la graine et l'ensemencement l'année suivante, ce qui permet de ne pas dépendre de l'achat annuel aux producteurs de semences (comme c'est le cas pour les hybrides du maïs et du tournesol).

- La résolution du problème des mauvaises herbes annuelles et pérennes est résolu avec trois opérations de pulvérisation d'un herbicide non sélectif (à base de glyphosate).

3. Herbicide à spectre large, qui affecte les autres cultures mais non pas le soja ayant le gène RR. 
RECHERCHES

Julio H. ELVERDÍN, Andrea MAGGIO, José MUCHNIK

Figure 2. Évolution de la surface semée des principales cultures dans la province de Buenos Aires

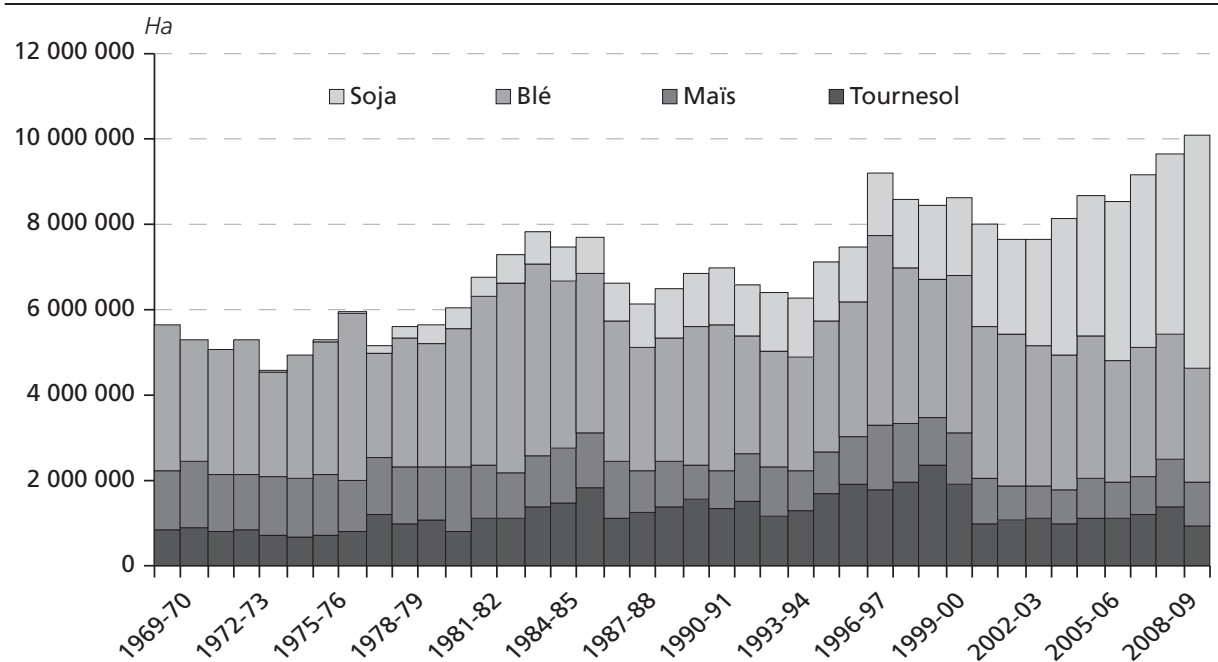

Source : Ministerio de Agricultura, Ganadería y Pesca de la Nación, 2010

Figure 3. Évolution du cheptel bovin dans la province de Buenos Aires

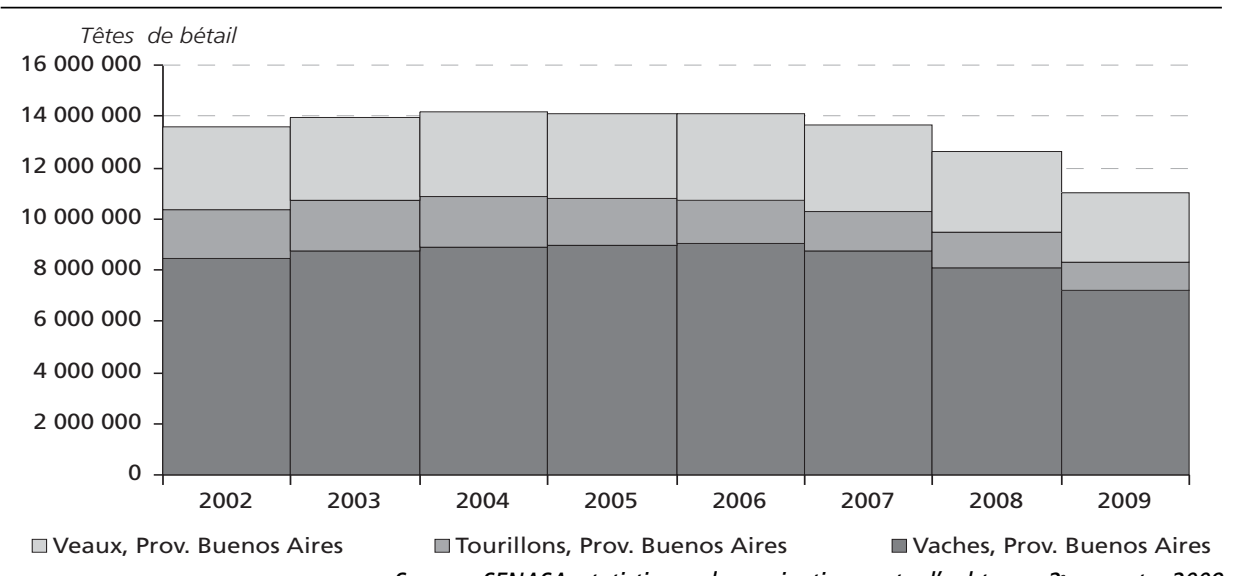

Source : SENASA, statistiques de vaccination contre l'aphteuse, $2^{\mathrm{e}}$ semestre 2009

- Le soja transgénique n'utilise pas plus de fertilisant que le soja traditionnel, mais il en faut moins que pour le tournesol et le maïs, qui deviennent ses concurrents en termes d'utilisation du sol du fait d'être ensemencés à la même époque.

- Le semis direct permet de réduire significativement le temps de travail opérationnel, d'économiser du carburant et de la maind'œuvre, et de réduire les risques d'érosion hydrique et éolienne.

- Le semis direct implique un fort investissement en machines agricoles, qui ne peut pas être toujours amorti par les producteurs, si bien que l'on assiste à la généralisation de la sous-traitance de services de machinerie agricole.

Les «pools de semis $(P S)$ » possèdent les caractéristiques suivantes :

- Des sociétés (fidéicommis, fonds d'investissement) qui se tournent vers la production agricole dans le but d'obtenir une rente des capitaux qu'elles investissent. Il s'agit de capitaux importants, n'appartenant pas à la filière agricole et souvent liés à la spéculation boursière. Ces groupes louent la terre et les machines agricoles pour la production. 
Ils gèrent également le capital, la terre et le travail des tiers, ainsi que la production et la commercialisation, en échange d'une redevance que les investisseurs doivent payer.

- Des modalités contractuelles parmi lesquelles prédomine le contrat «ponctuel » avec une valeur fixe à l'hectare ${ }^{4}$, dans le cadre duquel le pool décide de l'ensemble du processus de production.

- Une demande de grandes quantités d'intrants et de grands volumes de production, ce qui implique des transactions à grande échelle qui souvent excluent les opérateurs locaux (magasins de détail de commercialisation d'intrants agricoles, stockeurs, coopératives) et opèrent directement avec les grands fournisseurs d'intrants, l'industrie ou les exportateurs.

- Une ambiguïté dans le rapport des acteurs locaux à ces fonds d'investissement est ambigu. Souvent, ceux-ci ont permis à des producteurs, ayant loué leurs terres, de payer des dettes historiques à court terme et à beaucoup de sous-traitants ruraux de financer leurs investissements en machines agricoles. Par ailleurs, les producteurs et les institutions locales s'inquiètent des bénéfices à moyen et à long terme que peuvent apporter au territoire ces nouvelles modalités entrepreneuriales.

Le processus d'expansion du modèle de production basé sur le «STSD + PS » pose le problème et le besoin d'approfondir la recherche à plusieurs niveaux :

Au niveau écologique et environnemental : la prédominance à court terme d'une logique économique et financière de la gestion agricole risque de mettre en péril, à moyen terme, la reproduction des ressources naturelles et la durabilité environnemen-

4. Normalement libellés en quintaux de soja ou en dollars américains par hectare. Les montants payés par le business financier sont souvent supérieurs à ceux des producteurs. tale. Les dispositifs de recherche scientifique devraient par conséquent être renforcés pour déterminer l'impact sur : (i) la biodiversité, comme conséquence de l'utilisation massive d'herbicides à base de glyphosate ; (ii) la pollution de l'eau, causée par l'excès ou la mauvaise utilisation d'insecticides et de fertilisants; (iii) la structure et la fertilité des sols, en raison du manque de rotation des cultures et/ou d'une restitution insuffisante de nutriments ; (iv) l'extension des brûlis et l'élimination de forêts.

Au niveau des dynamiques territoriales: la diminution du nombre de familles habitant à la campagne, avec à la clé le dépeuplement et l'affaiblissement d'infrastructures déjà pauvres (logements, écoles, services), ainsi que l'émigration de professionnels et la perte de métiers ruraux sont autant de facteurs qui contribuent à fragiliser les sociétés rurales, favorisant les processus de délocalisation.

Au niveau de la sécurité alimentaire: étant donné qu'en Argentine le soja n'est pas consommé de manière directe et qu'il s'agit d'une culture qui déplace d'autres activités et d'autres cultures, il se pose la question de la réglementation de son expansion ainsi que des mécanismes techniques, économiques et législatifs à mettre en place en vue de protéger les autres productions (viande, lait, blé, riz, pomme de terre...) essentielles pour l'alimentation de la population.

La résilience du système: quel est l'avenir de ces systèmes de production, basés notamment sur une logique financière qui est à l'origine de productions à échelle croissante ? Est-il possible de maintenir une agriculture concentrée, avec un nombre réduit d'acteurs ? Peut-on développer le territoire rural et ses communautés à partir de ce modèle de production ? Quel rôle revient à la population rurale déplacée de ses activités agricoles? 


\section{Les stratégies des producteurs}

Dans le contexte décrit ci-dessus, de petits et moyens producteurs, ayant des caractéristiques structurelles, historiques et même familiales similaires ont emprunté des chemins très différents. Beaucoup d'entre eux ont été mis à l'écart de l'activité agricole, alors que d'autres résistent encore. Il s'agit de processus complexes où interviennent de variables multiples, que nous divisons en deux catégories : celles qui dépendent de l'environnement socio-territorial, et celles qui dérivent des conditions techniques et économiques (schéma 2).

Les stratégies les plus courantes, comme nous le verrons ensuite dans le cas de Bolívar, sont les suivantes :

Concentration et économies d'échelle. En louant des champs voisins le producteur met l'accent sur l'investissement en machinerie agricole, transformant son profil et l'essence de son travail de chacarero $^{5}$ en entrepreneur rural qui travaille avec les coopératives ou les stockeurs de céréales. Il intensifie l'élevage pour ne pas s'en défaire. Il réside dans son exploitation ou dans les centres urbains.

Élevage intensifié. Il s'agit de producteurs qui ne peuvent pas s'offrir la technologie agricole nécessaire et se tournent vers l'élevage. Ils proposent alors une intensification de leur activité. S'ils disposent d'une parcelle qui peut être cultivée, ils la mettent en fermage. En règle générale ils ne résident plus dans leur exploitation.

5. Ansaldi (1993) considère que le chacarero constitue une classe sociale spécifique du capitalisme agraire argentin, dont la caractéristique principale d'identification dans les premières décennies du $\mathrm{XX}^{\mathrm{e}}$ siècle était de ne pas être propriétaire de la terre travaillée. Ensuite, même s'ils ont accédé à la propriété, ils ont gardé cette référence identitaire chacarera, avec un mode de vie spécifique, d'agriculteurs familiaux, avec des aspirations d'accumulation capitaliste, et une vie sociale centrée sur le monde rural, l'agriculture et/ou l'élevage.
Fermage partiel. Une partie de la surface agricole est louée pour renforcer leur activité tournée vers un autre type de production. Cette stratégie s'applique aux producteurs de lait, de viande de porc, aux éleveurs et engraisseurs qui disposent de sols de bonne qualité et les utilisent pour cultiver du soja plutôt que des pâturages.

Reconversion en entrepreneurs sous-traitants ruraux. Les producteurs, qu'ils soient propriétaires ou non, se sont reconvertis en sous-traitants ruraux avec un réinvestissement important dans la machinerie agricole pour offrir différents services (labour et/ou ensemencement et/ou pulvérisation et/ou récolte et/ou production de meules ou ensilage...) à d'autres producteurs ou à des pools de semis. Les propriétaires, eux, mettent la totalité de leurs terres en fermage.

Reconversion avec intensification. Il s'agit de producteurs qui ont entrepris diverses activités plus intensives, essentiellement par rapport à la main-d'œuvre, car ils recherchent une filière de production plus rentable sur de petites surfaces (production de porcs, de fruits rouges, horticulture extensive, engraissement intensif de bovins, production de fromages et/ou de conserves...). Dans cette catégorie, figurent les « néo-ruraux », producteurs dont les revenus proviennent essentiellement d'une autre activité économique et qui cherchent à investir dans ce type de production non conventionnelle. En règle générale ces producteurs ne résident pas dans leur exploitation.

Rentiers. Il s'agit des personnes qui ont mis la totalité de leurs parcelles en fermage ou en métayage et n'interviennent à aucun moment dans le processus de production, si ce n'est pour fixer le montant du bail. Dans certains cas, ces personnes ont du bétail qui leur appartient sur les parcelles peu appropriées à la production agricole. La majorité d'entre eux habite dans des villes où ils développent d'autres activités économiques. 
Schéma 2. Variables intervenant dans la définition des stratégies des agricultures familiales

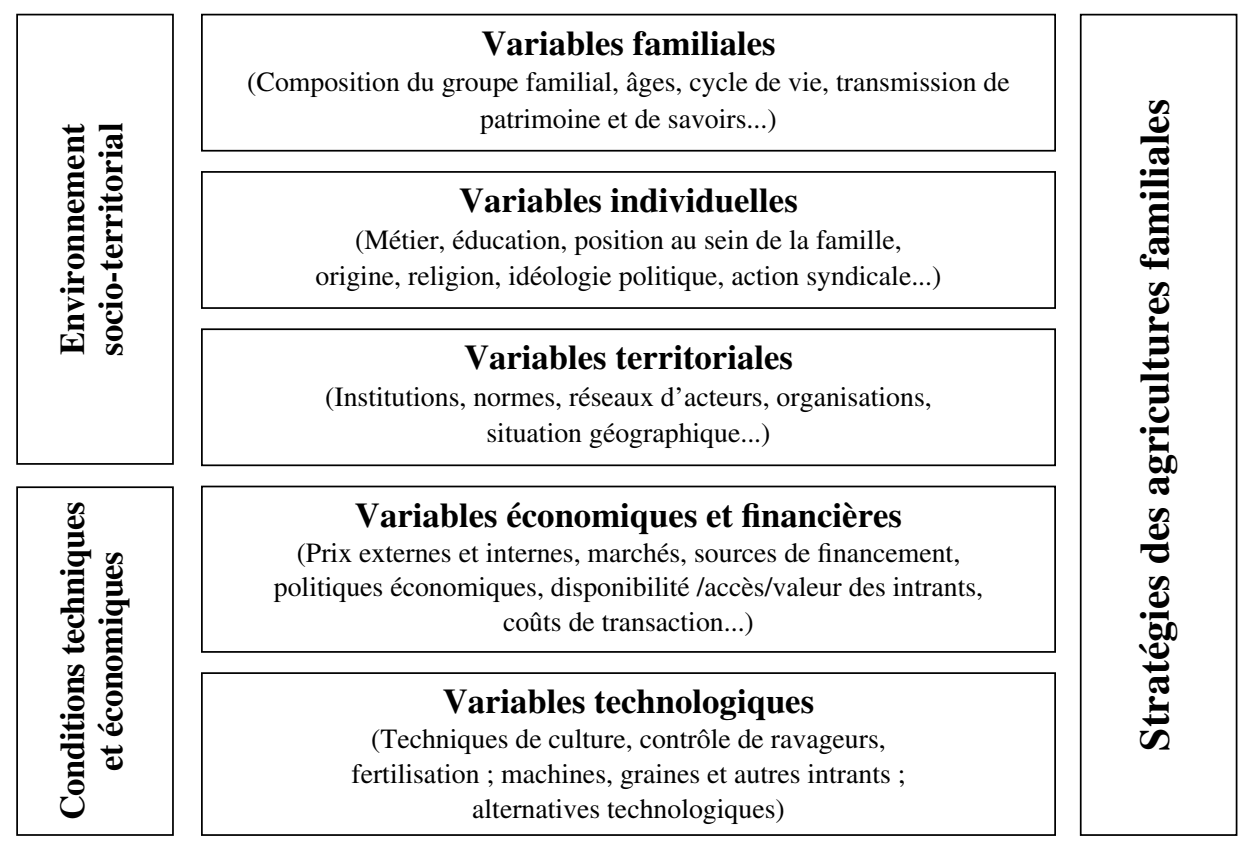

Sources : les auteurs

Dans tous les cas, les familles qui résident dans leur exploitation ont une relation étroite avec les centres urbains où elles trouvent la plupart des services publics et privés nécessaires (mécaniciens, consignataires, banques, médecins, commerces...).

Ce que nous constatons c'est que l'optimisation des bénéfices n'est pas l'unique objectif de la majorité des producteurs familiaux. Certaines études analysent le comportement de ces producteurs en prenant en considération la notion de risque (Iorio et Mosciaro, 2008), expliquent les motifs pour lesquels ils ne pratiquent pas la monoculture et montrent la façon dont ils donnent la priorité à l'assolement et au rôle de l'élevage dans les systèmes de production. Mais la présence croissante des pools de semis avec un fonctionnement fondé sur une logique financière pourrait faire en sorte que ces schémas (diversification, assolement...) se simplifient et que la tendance à la « sojisation » et à l'industrialisation agricole s'intensifie.

\section{Les dynamiques territoriales}

\section{Le territoire de Bolívar}

Pour analyser le rapport entre le processus d'industrialisation agricole et les dynamiques territoriales, nous nous appuierons sur la base d'entretiens réalisés auprès de producteurs familiaux de la localité de San Carlos de Bolívar. Nous utiliserons le concept de territoire en tant qu' « espace socialement construit, culturellement marqué et institutionnellement réglementé » (Lopez et al., 1997).

Nous avons choisi Bolívar ${ }^{6}$ car :

i) cette localité est représentative du processus de changement de la production agricole pampéenne que nous sommes en train d'analyser, comme nous pouvons les confirmer en comparant les figures let 2 ;

6. La commune de San Carlos de Bolívar est située au centre ouest de la province de Buenos Aires. Elle compte 32440 habitants, dont $12,2 \%$ de population rurale (Recensement national de population, 2001). 
Figure 4. Évolution de la surface semée des principales cultures dans le Partido* de Bolívar

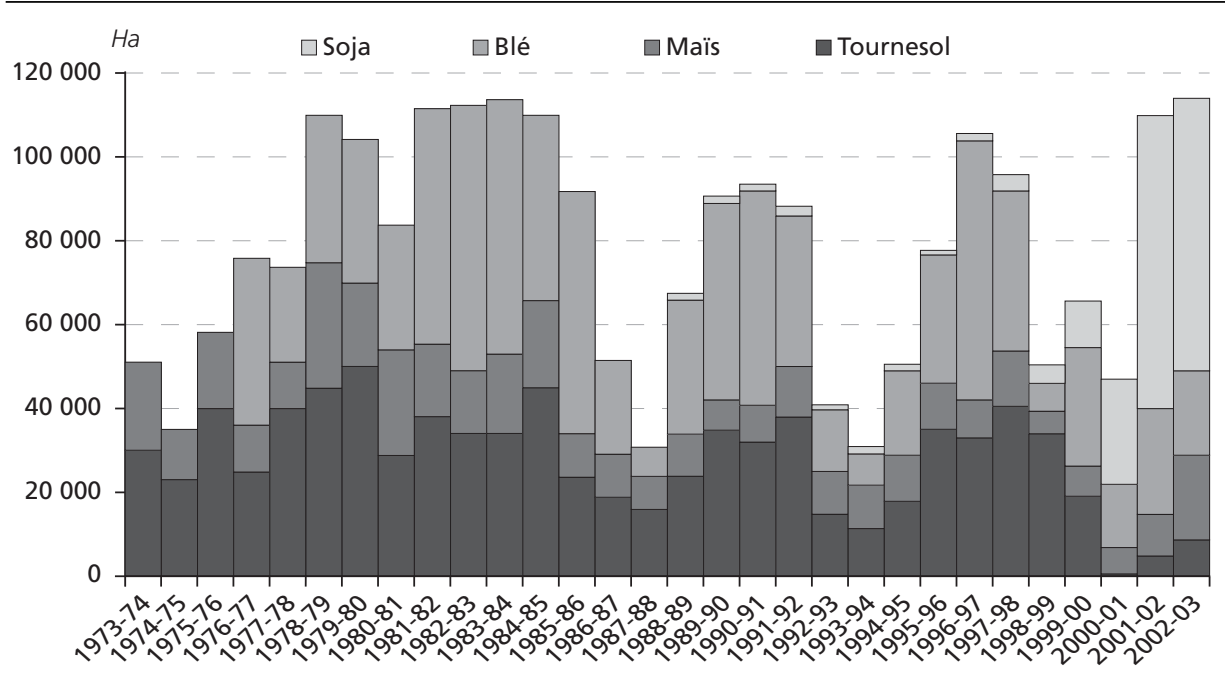

*Partido : Unité administrative qui regroupe plusieurs communes

Source : MAGYP, Délégation Bolívar, 2009

ii) il existe des données historiques et statistiques ;

iii) c'est une des localités de la province de Buenos Aires avec une proportion majeure des petits exploitants familiaux habitant dans la ferme ( $72 \%$ du total des exploitations, Obshatko, 2007).

Comme source d'information, nous nous sommes appuyés sur des entretiens réalisés auprès de producteurs familiaux. Cinquante-six unités familiales ont été enquêtées à partir des données cadastrales et productives. Dans le même secteur 16 enquêtes approfondies de producteurs familiaux ont été réalisées, ainsi que 15 enquêtes auprès de fonctionnaires, institutions ou représentants d'entités privées (Guber, 2004 ; Sirvent, 1999)

Depuis sa fondation en 1878, la communauté de Bolívar a prévu le développement de petites exploitations d'agriculture et d'élevage sur son territoire en intégrant des parcelles de 24 hectares, appelées chacras, dans l'espace entourant la ville. Puis en 1884 est créé un deuxième cordon de chacras de 84 ha et 141 ha. En tout, plus de 300 parcelles ont été créées. Avec le temps, elles ont été sous-divisées en unités plus petites, ce qui a, dans de nombreux cas, obligé la totalité ou une partie de la famille qui ne pouvait atteindre l'unité économique ${ }^{7}$ à émigrer vers les villes. Depuis les années 80 il est interdit de réaliser des sous-divisions inférieures à cette surface.

De plus, certaines parcelles sont nées de la division en lots de grandes propriétés qui, à la fin du XIX ${ }^{\mathrm{e}}$ siècle, étaient louées aux colons immigrants par l'intermédiaire de contrats précaires avec paiement sous forme de céréales et de provision de tous les produits domestiques nécessaires à la gestion de la propriété. Après la révolution chacarera dans la région de la pampa, connue sous le nom du Cri d'Alcorta, le Gouvernement national a facilité la vente de lots aux familles de colons qui les exploitaient. (Blanco, 2007). Aujourd'hui les familles se souviennent du moment où elles sont devenues propriétaires comme d'un moment charnière dans leur vie qui leur a permis de s'installer et de commencer à prospérer.

7. L'unité économique adoptée par le gouvernement provincial pour Bolívar est comprise entre 100 et 130 ha. Or avant cette date, à l'occasion de certaines sous-divisions l'on trouve des unités de 5 ha. (Source : INTA AER Bolívar, 2007) 


\section{Figure 5. Évolution du cheptel bovin et de ses principales catégories}

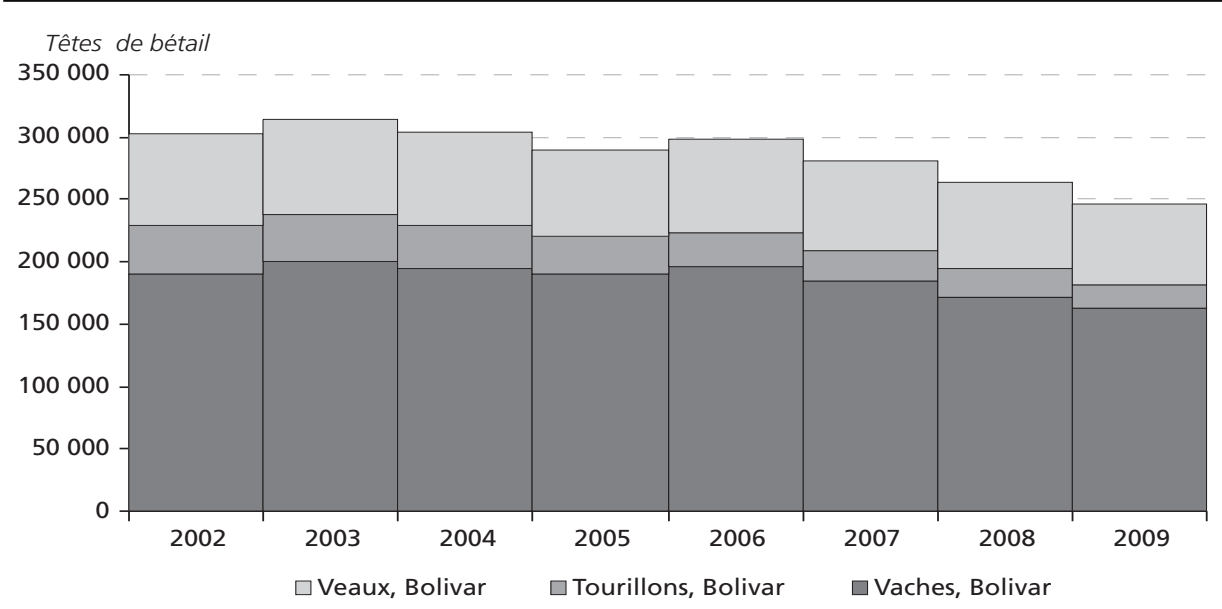

Source : SENASA, statistiques de vaccination contre l'aphteuse, 2emestre 2009

Un phénomène significatif pour l'analyse est de tenir compte que cette zone a souffert des inondations depuis la fin des années 1980 jusqu'à 2001 ; ceci a eu une grande influence sur les surfaces agricoles productives, favorisant le maintien de l'élevage pendant cette période.

\section{Les exploitations agricoles familiales}

Les chacras se rassemblent en hameaux où se déroulent les activités de production liées à l'agriculture et l'élevage, où elles ont développé un environnement socioculturel et productif particulier. Dans la plupart des cas, ces lieux forgent leur identité autour d'une école rurale, aujourd'hui encore parfois opérationnelle. C'est également là qu'ont vu le jour la coopérative ou le club rural qui propose des activités sociales ou sportives (dîners, danses, concours, matchs de football) (INTA AER Bolívar, 2007).

Le système de production qui prédomine à Bolívar est celui basé sur l'association de l'agriculture et de l'élevage bovin. L'évolution des exploitations confirme la tendance générale dans la région de la Pampa ( $c f$. supra schéma 2.).

La culture de soja démarre à Bolívar en 1998 et devient significative en 2001 une fois finies les inondations (figure 4). Elle représente en $200857 \%$ de la surface cultivée, tandis que le maïs et le blé n'atteignent que $18 \%$ chacun. Par ailleurs le total de la surface agricole cultivée a augmenté de $20 \%$ au cours de cette période.

L'élevage à Bolívar a reculé de manière sensible à partir de l'année 2007, de manière cohérente avec l'évolution au niveau provincial, même s'il reste parmi les principales localités d'élevage de la province de Buenos Aires (figure 5). La plupart des familles enquêtées gardent leur cheptel, avec une conduite plus intensive.

Dans la même chacra se développe plus d'une activité productive, avec une dynamique de changement importante conditionnée par les fluctuations de l'économie, les rapports produit/intrant, la disponibilité de main-d'œuvre familiale et les conditions climatiques conjoncturelles (INTA AER Bolívar, 2008). On peut également y ajouter des activités qui ont lieu en dehors de l'exploitation comme les travaux en sous-traitance ou la fourniture de services agricoles (INTA AER Bolívar, 2007).

De nombreuses familles chacareras sont enracinées et habitent en milieu rural pour des raisons culturelles et aussi en raison des types de production intensive choisis qui requièrent une attention permanente (viande 
de porc et de lait, horticulture, apiculture). Elles conservent dans de rares cas, la production pour l'autoconsommation, à l'exception de quelques brebis ou cochons.

Tous leurs enfants sont scolarisés et ils se sacrifient pour leur offrir des études universitaires, bien qu'ils continuent à leur transmettre leurs savoir-faire dans le domaine de la production (INTA CRBAN, 2005).

Par ailleurs, le CNA 2002 indique que la moitié de la surface agricole de la localité est intégralement exploitée par ses propriétaires ou des membres de leur famille et que l'autre moitié combine la production propre avec des parcelles en fermage ou sous contrat ponctuel, seulement $8 \%$ des exploitations ont été cédées en fermage dans leur totalité. On sait toutefois que cette tendance a augmenté ces dernières années, tout comme dans le reste de la région. Plusieurs personnes interrogées ont affirmé que la majorité des petits et moyens producteurs qui ont mis en fermage leurs champs pour y cultiver du soja ont pu, aux cinq dernières campagnes, éponger leurs dettes financières et seraient aujourd'hui à même de reprendre leur propre production en faisant appel à des prestataires de services.

Ces producteurs gèrent leurs facteurs de production (la terre, la main-d'œuvre et le capital) en fonction d'une logique commerciale. L'un des producteurs interrogés a déclaré : "Nous n'exploitons pas la terre car nous la considérons comme un bien qu'il faut qu'il produise avec la même efficacité qu'une entreprise, dans notre cas, une entreprise familiale. »

On peut affirmer que lorsqu'il s'agit de vendre leurs produits ils ne sont pas liés à un réseau de commercialisation en particulier puisque, au contraire, ils recherchent en permanence de meilleures conditions de vente.

À en croire les entretiens, il existe très peu de lieux pour les échanges professionnels. Par contre les rencontres culturelles et de loisirs, dans l'espace commun qu'est l'école, ne manquent pas. Toutefois, l'an dernier, à l'issue du « conflit agraire », des espaces ont été créés pour organiser des rassemblements de producteurs où chacun peut débattre des positions à adopter face aux propositions du Gouvernement et des associations professionnelles.

\section{Apparition de nouveaux acteurs : vers une nouvelle ruralité}

Étant donné l'intérêt des investisseurs qui n'appartiennent pas au secteur de l'agriculture et de l'élevage et l'utilisation massive de l'Internet et de la téléphonie mobile, de nouveaux acteurs, jusqu'alors étrangers à cette activité, sont apparus, tels que les agents immobiliers pour la vente et la location de terrains ; les vendeurs d'intrants à distance ; de nouveaux entrepreneurs venant de la ville ; des professionnels du tourisme rural.

De même, les opérateurs, les prestataires et les fournisseurs professionnels qui travaillent avec les pools de semis viennent de différentes zones de la région sans habiter nécessairement dans la commune ou interagir avec elle.

À l'instar des nouveaux acteurs, les familles «chacareras » ont elles aussi façonné une nouvelle identité territoriale, très différente de l'ancienne image du colon immigrant qui travaillait pour son patron. Les caractéristiques intrinsèques et les externalités des modèles de gestion et de production qui sont en train de s'imposer depuis quelques années favorisent le développement d'un type de producteur professionnalisé, qui considère la terre comme un support, un instrument, de son travail plutôt que comme un lieu de résidence (Albaladejo, 2004).

\section{Conclusion}

En premier lieu, nous constatons, à la lumière des données quantitatives relevées, qu'une forte tendance au « tout soja » et à l'industrialisation agricole s'impose dans la région de la Pampa. Il est essentiel de 
renforcer les dispositifs de recherche scientifique afin de pouvoir évaluer objectivement son impact sur l'environnement, sur la dynamique des sociétés rurales et sur la sécurité alimentaire de la population.

Associés à cette évolution technicoéconomique des systèmes de production, se développent des changements qualitatifs dans les sociétés rurales et dans les dynamiques territoriales, accentuant la tendance d'affaiblissement (pendant les trente dernières années) des tissus sociaux dans les villages et hameaux en milieu rural au profit des centres urbains proches (agro-villes).

Parallèlement à la tendance prédominante, il y a un «contre-courant » qui reflète la diversité des stratégies mises en place par les producteurs, en particulier de petits et moyens exploitants, qui ont tendance à associer le soja avec des activités ancrées dans le territoire et plus significatives du point de vue de leur métier et de leurs identités professionnelles. Par conséquent, loin d'avoir un espace rural uniforme, nous nous trouvons face à une combinatoire d'activités avec différents degrés d'ancrage territorial.

Du point de vue conceptuel, on devrait se poser la question de savoir quels sont les outils et les approches les plus adaptés pour analyser cette situation. Les approches traditionnelles en termes de filières et d'agribusiness peuvent aider à comprendre les rapports entre les différents acteurs du secteur de la production de soja ou d'autres céréales mais présentent d'importantes lacunes pour expliquer l'évolution des activités liées à la production territoriale. Nous pourrions alors nous interroger sur la pertinence de l'approche en termes de systèmes agroalimentaires localisés (Syal) comme outil pour analyser les situations où, paradoxalement, la tendance principale est à la délocalisation. Dans la mesure où l'approche Syal part des dynamiques territoriales, de l'identification et de la spécification des ressources du territoire, elle pourrait s'avérer intéressante pour comprendre la persistance des contre-courants qui concernent les activités articulées autour de stratégies visant à minimiser les risques, moins dépendantes des fluctuations des marchés d'exportation et plus difficilement délocalisables.

Du point de vue opérationnel, nous nous trouvons face à un dilemme semblable puisque traditionnellement les instruments politiques de régulation sont définis selon les filières structurées par produit. Or la vie des sociétés rurales et les activités de l'ensemble du territoire exigent d'articuler les dynamiques territoriales et les filières de production, ce qui impliquerait le développement de nouvelles articulations entre différents dispositifs institutionnels et niveaux administratifs (national, territorial, provincial, régional et municipal). 


\section{RÉFÉRENCES BIBLIOGRAPHIQUES}

Albaladejo C. (2004). Innovaciones discretas y reterritorialización de la actividad agropecuaria en Argentina, Brasil y Francia. In Albaladejo C., Bustos Cara R. (éds.), «Desarrollo local y nuevas ruralidades en Argentina/Développement local et multifonctionnalité des territoires ruraux en Argentine ». Bahía Blanca, 412 p.

Ansaldi W. (1993). Cité par Balsa J. El desvanecimiento del mundo chacarero : transformaciones sociales en la agricultura bonaerense : 1937-1988.

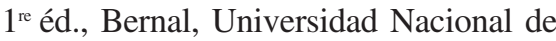
Quilmes, 2006. 328 p.

Blanco M. (2007). Reforma en el agro pampeano. Arrendamiento, propiedad y legislación agraria en la provincia de Buenos Aires. 1940-1960. Buenos Aires, $1^{\text {re }}$ éd., Bernal, Universidad Nacional de Quilmes, 350 p.

Berger S. (2006). Made in Monde. Paris, Seuil, $357 \mathrm{p}$.

Champredonde M. (2008). Localización, deslocalización, ¿relocalización? de la calidad de las carnes vacunas pampeanas argentinas. Impacto territorial. In Velarde I., Maggio A., Otero J. (ed. INTA) "Los sistemas agroalimentarios localizados en Argentina", Buenos Aires, $183 \mathrm{p}$.

INDEC. CNA 1988 (Censo Nacional Agropecuario del año 1988) (1992). Resultados Generales : Provincia de Buenos Aires. Buenos Aires, Instituto Nacional de Estadística y Censos, 152 p. INDEC. CNA 2002 (Censo Nacional Agropecuario del año 2002) (2008). Resultados de Provincia de Buenos Aires. Buenos Aires, Instituto Nacional de Estadística y Censos, www.indec.mecon.ar INDEC. CNA 2008 (Censo Nacional Agropecuario del año 2008) (2009). Resultados provisorios. Instituto Nacional de Estadística y Censos, Buenos Aires, www.indec.mecon.ar
Fischler C. (1990). L'Homnivore, Paris, Odile Jacob.

Giberti H. (1970). Historia económica de la ganadería argentina. Buenos Aires, Hyspamérica, 275 p.

Guber R. (2004). El salvaje metropolitano : reconstrucción del conocimiento social en el trabajo de campo Buenos Aires, $1^{\circ}$ edición. : Paidós Estudios de Comunicación. INTA AER Bolívar. (2007). PROFAM PreProyecto Promoción de la pequeña agricultura familiar en Bolívar para su desarrollo y bienestar. INTA, Agencia de Extensión Rural Bolívar.

INTA AER Bolívar. (2008). Informes para comunic@r $N^{o} 165$ y 167. INTA, Agencia de Extensión Rural Bolívar.

INTA CRBAN (2005). Desarrollo Local en Buenos Aires Norte-Algunas características del Territorio. INTA, Pergamino, Mimeo.

Iorio C., Mosciaro M. (2008). Diversificación o monocultura en el sudeste bonaerense : aportes desde una perspectiva económica. Buenos Aires, Revista Argentina de Economía Agraria, Edición especial, Otoño, 20 p.

Lopez E., Muchnik J., (éd.) (1997). Petites entreprises et grands enjeux : le développement agroalimentaire local. Paris, L'Harmattan, 2 tomes, 716 p.

Mauss M. (1935). Les techniques du corps. Anthropologie et Sociologie. Paris, 1980, PUF.

MAGYP Ministerio de Agricultura, Ganadería y Pesca de la Nación. (2010). Sistema integrado de información agropecuaria. Series y Estadísticas, Buenos Aires, http://www.minagri.gob.ar. Moity-Maïzi P., Muchnik J. (2005). Circulation et construction de savoirfaire : questions pour une anthropologie des systèmes agroalimentaires localisés. Revue Industries Alimentaires et Agricoles., décembre, p. 16-25. 
Muchnik J., Sanz-Cañada J., Torres Tizon Ph. (1996). Qu'est-ce que le territoire. Salcido G. (2008). Systèmes agroalimentaires localisés : état des recherches et perspectives. Cahiers d'Agriculture, vol. $17, \mathrm{n}^{\circ} 6$.

Obschatko E., Foti M., Román M. (2007). Estudio de la importancia de los pequeños productores agropecuarios en la producción agropecuaria y en el empleo en base al censo nacional agropecuario 2002. Buenos Aires, lica-Proinder-Sagpya, $110 \mathrm{p}$.

Sirvent M.-T. (1999). Cultura popular y participación social. Una investigación en el barrio de Mataderos. Buenos Aires, Niño y Dávila Editores, Buenos AiresMadrid. In Di Meo G. (dir.) « les territoires du quotidien » Paris, L'Harmattan, p. 17-34 Tosi J. (2003). Diez años de cultivos de cosecha gruesa en el sudeste. INTA, Balcarce, Suplemento Económico, Visión Rural, $\mathrm{n}^{\circ} 50,7 \mathrm{p}$.

Tosi J. (2007a). Alternativas para cultivos invernales. INTA, Balcarce, Suplemento Económico, Visión Rural, n ${ }^{\circ}$ 67, 7 p.

Tosi J. (2007b). La producción ganadera: ¿Una lucha desigual? INTA, Balcarce, Suplemento Económico, Visión Rural, $\mathrm{n}^{\circ} 69,7 \mathrm{p}$.

Vanistendael S., Lecomte J. (2000) Le bonheur est toujours possible. Construire la résilience. Paris, Bayard. 